\title{
ON A MINIMAX INEQUALITY OF KY FAN
}

\author{
CHUNG-WEI HA \\ Dedicated to Professor Ky Fan on his 7Oth birthday
}

\begin{abstract}
An extension of a minimax inequality of Ky Fan is given. It is then used to generalize to the set-valued setting a fixed point theorem of Ky Fan.
\end{abstract}

Since Fan gave his minimax inequality in [4], various extensions of this interesting result have been obtained (see $[\mathbf{5}]$ and the references there). One was proved in $[\mathbf{6}$, Theorem 3], which can be formulated in the following form:

THEOREM 1. Let $E, F$ be Hausdorff topological vector spaces, $X \subset E, Y \subset F$ be nonempty convex subsets, $Y$ be compact. Let $f$ be a real-valued function defined on $X \times Y$ such that

(a) for each $y \in Y, f(x, y)$ is quasiconvex in $x \in X$;

(b) for each $x \in X, f(x, y)$ is upper semicontinuous in $y \in Y$.

If $T$ is a upper semicontinuous set-valued map defined on $X$ such that $T x$ is a nonempty closed convex subset of $Y$ for each $x \in X$, then

$$
\inf _{y \in T x} f(x, y) \leq \max _{y \in Y} \inf _{x \in X} f(x, y) \text {. }
$$

When $X=Y$ and the set-valued map $T$ is defined by $T x=\{x\}$ for $x \in X$, Theorem 1 reduces to Fan's manimax inequality [4, Theorem 1$]$. We recall that a real-valued function $\phi$ defined on a convex set $X$ is called quasiconvex if, for each real number $t$, the set $\{x \in X: \phi(x)<t\}$ is convex. Let $X, Y$ be topological spaces. By a set-valued map $T$ defined on $X$ with values in $Y$, we mean a map $T$ that assigns to each $x \in X$ a unique nonempty subset $T x$ of $Y$. T is called upper semicontinuous on $X$ if, for each open subset $G$ of $Y$, the set $\{x \in X: T x \subset G\}$ is open in $X$. It is easy to show (e.g. $[\mathbf{1}$, p. 72]) that if $Y$ is compact Hausdorff and if $T x$ is closed for each $x \in X$, then $T$ is upper semicontinuous on $X$ if and only if the graph $\{(x, y) \in X \times Y: y \in T x\}$ of $T$ is closed in $X \times Y$.

In [4] Fan also gave a version of the minimax inequality with a lopsided saddle point (see [4, Corollary 1]) which is useful in many situations. The main result of this note is the following Theorem 2, which is a variant of Theorem 1 with a lopsided saddle point.

THEOREM 2. Let $E, F$ be Hausdorff topological vector spaces, $X \subset E, Y \subset F$ be nonempty compact convex sets. If $g$ is a continuous real-valued function defined on $X \times Y$, and if $T$ is an upper semicontinuous set-valued map defined on $X$ such that

(c) for each $y \in Y, g(x, y)$ is quasiconvex in $x \in X$;

Received by the editors September 27, 1984 and, in revised form, February 3, 1986.

1980 Mathematics Subject Classification (1985 Revision). Primary 47H10; Secondary 49A29. 
(d) for each $x \in X, T x$ is a nonempty closed convex subset of $Y$, then there exist $x_{0} \in X$ and $y_{0} \in T x_{0}$ satisfying

$$
g\left(x_{0}, y_{0}\right) \leq g\left(x, y_{0}\right)
$$

for all $x \in X$.

ProOF. We define a real-valued function on $X \times Y$ by

$$
f(x, y)=g(x, y)-\min _{z \in X} g(z, y) .
$$

Clearly $f$ satisfies condition (a) in Theorem 1 . Moreover, it is easy to show (e.g. [1, p. 70]) that $f$ is continuous on $X \times Y$. By the compactness of $X$, for each $y \in Y$ there exists $x \in X$ such that $f(x, y)=0$ and hence, by Theorem 1 ,

$$
\inf _{y \in T x} f(x, y) \leq 0 .
$$

Since the graph of $T$ is closed and so is compact in $X \times Y$, the infimum in (2) is attained. The result now follows.

As an application, we use Theorem 2 to prove the following Theorem 3 , which is a generalization to the set-valued setting of Fan's fixed point theorem [3, Theorem 1].

THEOREM 3. Let $E$ be a locally convex Hausdorff topological vector space, $X \subset$ $E$ be a nonempty compact convex subset. Let $T$ be an upper semicontinuous setvalued map defined on $X$ such that $T x$ is a compact convex subset of $E$ for each $x \in X$. Then either $T$ has a fixed point in $X$, or there exist $x_{0} \in X, u_{0} \in T x_{0}$, and a continuous seminorm $p$ on $E$ such that

$$
0<p\left(x_{0}-u_{0}\right) \leq p\left(x-u_{0}\right)
$$

for all $x \in X$.

Proof. Suppose that $T$ has no fixed point in $X$. Then for each $x \in X$, the origin 0 of $E$ does not belong to the compact set $x-T x$ and so there exist $\delta_{x}>0$ and a continuous seminorm $p_{x}$ on $E$ such that $p_{x}(x-u)>2 \delta_{x}$ for all $u \in T x$. By the upper semicontinuity of $T$, there exists an open neighborhood $N_{x}$ of $x$ in $X$ such that $p_{x}(z-v)>\delta_{x}$ for all $z \in N_{x}$ and $v \in T z$. Since the family $\left\{N_{x}: x \in X\right\}$ is an open cover of the compact set $X$, there exists a finite subset $\left\{x_{1}, \ldots, x_{m}\right\}$ of $X$ such that $\left\{N_{x_{i}}: 1 \leq i \leq m\right\}$ covers $X$. Let $p=\max \left\{p_{x_{i}}: 1 \leq i \leq m\right\}$ and $\delta=\min \left\{\delta_{x_{i}}: 1 \leq i \leq m\right\}>0$. Then $p$ is a continuous seminorm on $E$ and

$$
p(x-u)>\delta
$$

for all $x \in X$ and $u \in T x$.

It can be shown (e.g. [1, p. 72]) that the image $T(X)=\bigcup\{T x \subset Y: x \in X\}$ of $T$ is compact. As the assumptions on $X$ and $T$ remain unchanged in the completion of $E$, without loss of generality we can assume that $E$ is complete. Let $Y$ be the closed convex hull of the image $T(X)$ of $T$ in $E$, then $Y$ is compact convex. We define a real-valued function $g$ on $X \times Y$ by $g(x, y)=p(x-y)$. Clearly $g$ and $T$ satisfy the hypothesis of Theorem 2. (3) now follows from (1) and (4). 
As a direct consequence of Theorem 3, we have the following

THEOREM 4. Let $E$ be a locally convex Hausdorff topological vector space, $X \subset$ $E$ be a nonempty compact convex subset. Let $T$ be an upper semicontinuous setvalued map defined on $X$ such that $T x$ is a compact convex subset of $E$ for each $x \in X$. If $T$ satisfies the following condition:

(e) for any $x \in X, u \in T x$, there exists a number $\lambda$ (real or complex, depending on whether the vector space $E$ is real or complex) such that

$$
|\lambda|<1 \text { and } \lambda x+(1-\lambda) u \in X
$$

then $T$ has a fixed point in $X$.

Proof. Suppose that $T$ has no fixed point in $X$. By Theorem 3 , there exist $x_{0} \in X, u_{0} \in T x_{0}$ and a continuous seminorm $p$ on $E$ such that (3) holds. For these $x_{0}$ and $u_{0} \in T x_{0}$, by (e) there exists a number $\lambda$ such that $|\lambda|<1$ and $x=\lambda x_{0}+(1-\lambda) u_{0} \in X$. Then

$$
0<p\left(x_{0}-u_{0}\right) \leq|\lambda| p\left(x_{0}-u_{0}\right),
$$

which contradicts $|\lambda|<1$.

Theorem 4 generalizes to the set-valued setting Fan's result [3, Theorem 3]. It is new only when the vector space $E$ is complex and the number $\lambda$ in the condition (e) is allowed to be complex. If the vector space $E$ is real, more general results have been obtained (see $[4,5]$ ). When the image $T(X)$ of $T$ is contained in $X$, the condition (e) is trivially satisfied and Theorem 4 reduces to the well-known fixed point theorem of Fan [2, Theorem 1]. An extension of [3, Theorem 3] was proved under a stronger hypothesis in [7], which does not contain Fan's fixed point theorem [2, Theorem 1].

\section{REFERENCES}

1. J. P. Aubin, Mathematical methods of game and economic theory, North-Holland, Amsterdam, 1979.

2. K. Fan, Fixed-point and minimax theorems in locally convex topological linear spaces, Proc. Nat. Acad. Sci. U.S.A. 38 (1952), 121-126.

3. K. Fan, Extensions of two fixed point theorems of F. E. Browder, Math. Z. 112 (1969), 234-240.

4. K. Fan, A minimax inequality and applications, Inequalities, vol. III, Academic Press, New York and London, 1972, pp. 103-113.

5. K. Fan, Some properties of convex sets realted to fixed point theorems, Math. Ann 266 (1984), 519-537.

6. C.-W. Ha, Minimax and fixed point theorems, Math. Ann. 248 (1980), 73-77.

7. S. Reich, Fixed points in locally convex spaces, Math. Z. 125 (1972), 17-31.

Department of Mathematics, National Tsing hua University, Hsinchu, TAIWAN, R.O.C. 